\title{
HCPA 48 ANOS: DESTAQUE NA PRODUÇÃO CIENTÍFICA
}

\author{
48 YEARS OF HCPA: PROMINENCE IN ACADEMIC \\ RESEARCH
}

\author{
Cristina Haeffner ${ }^{1}$, Sonia R. Zanotto², Jorge Almeida Guimarães ${ }^{3}$
}

Clin Biomed Res. 2019;39(4):266-278

1 Biblioteca do Instituto de Educação e Pesquisa (IEP), Hospital Moinhos de Vento. Porto Alegre, RS, Brasil.

2 Instituto Brasileiro de Geografia e Estatística (IBGE - RS). Porto Alegre, RS, Brasil.

3 Hospital de Clinicas de Porto Alegre (HCPA) e Centro de Biotecnologia, Universidade Federal do Rio Grande do Sul (UFRGS). Porto Alegre, RS, Brasil.

Autor correspondente: Jorge Almeida Guimarães jorgeguimaraes@hcpa.edu.br Hospital de Clínicas de Porto Alegre (HCPA)

Ramiro Barcelos, 2350. 90035-903, Porto Alegre, RS, Brasil.

\section{RESUMO}

Este artigo ilustra o desenvolvimento das atividades de pesquisa ao longo dos 48 anos do Hospital de Clínicas de Porto Alegre (HCPA). Fundado em 1971, o HCPA começa a atuar em pesquisa em 1974, data dos primeiros artigos indexados nas bases internacionais. O estudo abrange um período de 44 anos, de 1974 a 2018, para o qual foram levantados os artigos científicos publicados em periódicos indexados na base Web of Science por pesquisadores do próprio hospital, da Faculdade de Medicina e de outras unidades da UFRGS associados aos projetos de pesquisa do HCPA. O artigo resulta de um estudo cientométrico, sendo para tanto realizadas análises de indicadores bibliográficos, como produção total de artigos científicos experimentais, artigos de revisão e artigos completos em eventos (proceedings papers). Para facilidade de informação, os termos utilizados pelas bases foram mantidos em inglês ao longo do texto e nas tabelas e figuras. Um total de 6.383 documentos desses três tipos de artigos foi publicado no período. Mostra-se também a evolução temporal dessa produção. Foram ainda levantadas as principais áreas de pesquisa, as palavraschave dos artigos, as revistas, a colaboração internacional e menção aos trabalhos mais citados. Ao longo do estudo foi possível verificar que alguns pesquisadores, autores de trabalhos significativos, não indicam nos artigos sua vinculação ao HCPA e recomenda-se uma orientação do GPPG sobre o assunto.

Palavras-chave: Produção científica; cientometria; HCPA; análise bibliométrica

\section{ABSTRACT}

This article illustrates the development of research activities over the 48 years of the Hospital de Clínicas de Porto Alegre (HCPA). Founded in 1971, the HCPA begins its research activities in 1974, date of the first articles published in indexed journals. The study covers a period of 44 years, from 1974 to 2018 for which the scientific articles in journals indexed in the Web of Science database, published by researchers of the hospital including the Faculty of Medicine and other UFRGS units associated with the research projects of the HCPA were considered for this study. The article results from a scientometric study, and for this purpose analyzes of bibliographic indicators were carried out, covering: total production of experimental scientific articles, review articles and complete papers published in the proceedings of scientific events. For ease of information, the terms used by the bases were maintained in English throughout the text and in the tables and figures. A total of 6,383 documents of these three types of articles were published in the period. The temporal evolution of this production is also shown. The main research areas, the keywords of the articles, the journals, the international collaboration and the most cited papers were also raised. It was identified at the end that several authors though working in the hospital, do not indicate in the articles their linkage to the HCPA and thus, it is recommended to GPPG to develop some orientation to the researchers concerning to this subject.

Keywords: Scientific production; scientometrics; HCPA; bibliometric analysis

O Hospital de Clínicas de Porto Alegre (HCPA) foi criado como Empresa Pública de Direito Privado pela Lei 5.640, de 2 de setembro de 1970, sendo vinculado funcional e diretamente ao Ministério da Educação. Seu Estatuto foi publicado no Diário Oficial da União em 19 de julho de 1971, data que 
marca o efetivo início das atividades da instituição'. Assim, em julho de 2021, o HCPA completará 50 anos. Com vinculação acadêmica à Universidade Federal do Rio Grande do Sul (UFRGS) e, portanto, na condição de hospital universitário é uma instituição sui generis no Brasil, tendo sua governança garantida por autonomia financeira e gerencial asseguradas por legislação específica.

Com 833 leitos, entre unidades de internação, centros de tratamento intensivo e emergência (majoritariamente reservados ao SUS) e em franca expansão, é amplamente reconhecida a trajetória do HCPA no exercício da assistência médica para pleno atendimento hospitalar, constituindo um complexo laboratorial e de assistência médicohospitalar, vale dizer no largo escopo da proteção à saúde, cobrindo toda a escala de especialidades e serviços, aí incluído um exemplar serviço de enfermagem, além de outros. Parte importante do sucesso do HCPA se deve à continuidade administrativa. Ao longo dos seus 48 anos, o HCPA foi administrado por apenas sete Presidentes: Dr. Milton Dias: 1971-1976; Dr. Mário Rangel Ballvé: 1976-1980; Dr. Loreno Brentano: 19801984; Dr. Carlos César de Albuquerque: 19841996; Dr. Sergio Pinto Machado: 1996-2008 e Dr. Amarílio Vieira de Macedo Neto: 2008-2016. Para o período 2016-2020, a Presidência do HCPA está a cargo da Dra. Nadine Clausell.

Ao lado da atuação como hospital modelo no Brasil, o HCPA tem destacada atuação em pesquisa, abrangendo praticamente toda a gama das áreas médicas e biomédicas. As atividades de pesquisa são desenvolvidas no Centro de Pesquisa Experimental (CPE) e no Centro de Pesquisa Clínica (CPC), ambos vinculados ao Grupo de Pesquisa e Pós-Graduação (GPPG), responsável institucional por essas atividades. Nessa estrutura de pesquisa estão sediados seis Institutos Nacionais de Ciência e Tecnologia (INCTs) dentre os 252 inicialmente selecionados nacionalmente para financiamento pelo $\mathrm{CNPq}$ e agências participantes do consórcio INCT (CAPES, FINEP e Fundações Estaduais de Amparo à Pesquisa). Da mesma forma, os demais grupos de pesquisa também estão vinculados ao GPPG, que dá também suporte à pesquisa a 22 Programas de Pós-Graduação (Mestrado e Doutorado) da UFRGS e a dois mestrados profissionais próprios do HCPA.

Este artigo ilustra o desenvolvimento das atividades de pesquisa realizadas a partir de 1974 a 2018 , ou seja, ao longo de 44 dos 48 anos do HCPA. $O$ estudo toma como base os artigos científicos publicados em periódicos indexados na base Web of Science por pesquisadores do próprio hospital, da Faculdade de Medicina da UFRGS e outros associados aos projetos de pesquisa do HCPA.

\section{PROCEDIMENTOS METODOLÓGICOS}

O estudo abrange um período de 44 anos (1974 a 2018), para o qual foram levantadas as informações sobre as publicações científicas disponibilizadas nas bases de dados internacionais. O período anterior, de 1971 a 1973, foi dedicado ao início das atividades do hospital como unidade de atendimento médicohospitalar'.

$\mathrm{O}$ artigo resulta de um estudo cientométrico, sendo para tanto realizadas análises de indicadores bibliográficos extraídos de bases de dados internacionais reconhecidas, descritas a seguir. As análises foram realizadas através do levantamento na base Web of Science Core Collection, uma das maiores e mais completas bases de dados on-line pertencente atualmente à Clarivate Analytics (Philadelphia, USA) ${ }^{3}$. Para facilidade de informação, os termos utilizados pelas bases foram mantidos em inglês ao longo do texto e nas tabelas e figuras.

Procedeu-se a busca no campo de pesquisa avançada utilizando o rótulo $\mathrm{OO}$ (Organization), com as seguintes descrições: Hosp Clinicas Porto Alegre OR Clin Hosp Porto Alegre OR Hosp Clin Porto Alegre OR Clin Hosp CPE HCPA OR Porto Alegre Clin Hosp OR HCPA AND PORTO ALEGRE, para todos os documentos e também restringindo o tipo de documento como articles, reviews e proceedings papers. Os dados baixados foram trabalhados utilizando a planilha Excel.

Nas tabelas e outras ilustrações ao longo do texto, são apontadas as situações de dupla-contagem de artigos. A dupla-contagem ocorre sempre que os dados bibliométricos possibilitam a contagem de uma ou mais vezes de artigos como: na classificação das áreas do conhecimento; na análise individual, nos artigos em coautoria com participantes da mesma instituição; no caso de uma revisão ser também contabilizada pela base de dados como artigo completo e ainda, na cooperação entre autores de mais de uma instituição nacional ou internacional. Por outro lado, a análise das publicações nas revistas não incorpora o conceito de dupla-contagem, uma vez que um determinado artigo não é publicado em mais de uma única revista.

\section{RESULTADOS E DISCUSSÃO}

A Tabela 1 mostra que no período 1974 a 2018 a produção científica do HCPA atingiu um total de 8.730 documentos, publicações estas distribuídas entre os 13 tipos de documentos listados pela base Web of Science. Desse total, 6.383 (73\%) são as publicações objeto desse estudo: artigos científicos completos (5.674), artigos de revisão (563) e artigos completos em eventos científicos conhecidos como proceeding papers (146). Para efeito desse texto 
esses três tipos de publicações são, frequente e simplificadamente, denominados artigos. A Tabela 1 lista também os demais tipos de documentos, destacando-se resumos em congressos (1.607), cartas a editores (484), material editorial (204) e outros (52). Destaque-se que os números relativos ao total da Tabela incluem situações de dupla-contagem, em que eventualmente alguns abstracts, revisões, proceeding papers ou mesmo outros documentos tenham sido classificados pelas revistas como artigos completos. Computada a produção do HCPA em cada revista científica, onde não há dupla-contagem, o total real dessas publicações é de 6.630 artigos no período (ver Tabela 3).

A produção científica do HCPA vem tendo crescimento contínuo ao longo dos anos, como mostrado nos oito períodos indicados na Tabela 2, sendo esse crescimento substancialmente acentuado a partir de 2006, ou seja, nos últimos 13 anos. A Tabela 2 lista os dados quantitativos da produção científica geral e os artigos de revisão no período e a Figura 1 ilustra o crescimento desses indicadores. Verifica-se que a partir do quinquênio 2002 - 2006 essa evolução assume a característica de crescimento exponencial tanto para os artigos científicos propriamente ditos como para os artigos de revisão. Assim, a produção conjunta nos dois últimos quinquênios (2006-2010 e 2011-2015) e mais o triênio 2016-2018 representa $85 \%$ e $97 \%$ da produção total de todas as publicações e dos artigos de revisão, respectivamente.

Vale salientar que, entre os períodos 2006-2018, o crescimento dos artigos de revisão bibliográfica foi bem mais acentuado. Saindo de insignificantes números nos primeiros cinco quinquênios, para um total de 548 artigos atingido no período 2006-2018, representando um crescimento de 36,5 vezes em relação aos quinquênios anteriores, ou seja, mais de seis vezes o crescimento da produção de artigos científicos que teve crescimento de 5,8 vezes nos mesmos períodos.

Esse desempenho acentuado da produção de artigos de revisão a partir de 2002 é um padrão que se repete no desenvolvimento científico brasileiro em geral e tal desempenho coincide com a criação em 2001 do Portal de Periódicos da Capes. Desde 2001, o Portal de Periódicos da Capes passou a ser a ferramenta que permite aos pesquisadores brasileiros acessar o conteúdo científico mundial, possibilitando, portanto, a garantia de produzir revisões bibliográficas sem o risco de omitir importantes referências no tema em estudo. Os pesquisadores do HCPA têm, desde então, pleno acesso a essa importante ferramenta bibliográfica ${ }^{4}$.

As publicações do HCPA cobrem praticamente todas as áreas da medicina e das ciências biomédicas. A Tabela 3 lista as principais áreas, ou seja, aquelas em que foi publicado um mínimo de 50 artigos. Noutras 104 áreas foram publicados 908 artigos, perfazendo o total de 9.612 publicações no período 1974-2018, aí incluída a dupla-contagem de artigos classificados em mais de uma área. No conjunto, as 46 áreas da Tabela 3 (18\% do total de 252 áreas científicas da Web of Science) somam 8.704 publicações, ou seja, $90,5 \%$ do total de documentos, com grande destaque para as áreas de psiquiatria, neurociências, neurologia clínica e comportamento, que juntas respondem por 1.905 artigos $(19,8 \%)$ do total de publicações.

Tabela 1: Produção científica do Hospital de Clínicas de Porto Alegre 1974 a 2018

\begin{tabular}{|c|c|c|c|}
\hline N. & Tipos de documentos & $\mathrm{N}^{\circ}$ de documentos & $\%$ \\
\hline 1 & Article & 5.674 & 64,99 \\
\hline 2 & Review & 563 & 6,45 \\
\hline \multirow[t]{2}{*}{3} & Proceedings Paper & 146 & 1,67 \\
\hline & Sub-total & 6.383 & 73,12 \\
\hline 4 & Meeting Abstract & 1.607 & 18,41 \\
\hline 5 & Letter & 484 & 5,54 \\
\hline 6 & Editorial Material & 204 & 2,34 \\
\hline 7 & Correction & 25 & \\
\hline 8 & Note & 14 & \\
\hline 9 & Book Review & 9 & \\
\hline 10 & Discussion & 1 & 0,60 \\
\hline 11 & Book Chapter & 1 & \\
\hline 12 & Data Paper & 1 & \\
\hline \multirow[t]{2}{*}{13} & Retraction & 1 & \\
\hline & Total* & $8.730 *$ & 100 \\
\hline
\end{tabular}

*Dados incluem dupla-contagem de documentos que compõem os dados da Tabela. 
Tabela 2: Crescimento da produção científica do HCPA nos oito quinquênios (1974-2015) e triênio 2016-2018. Número de Documentos Científicos e de Artigos de Revisão.

\begin{tabular}{ccc}
\hline Anos & Número de documentos & Revisões \\
\hline $1974-1985$ & 25 & 2 \\
$1986-1990$ & 45 & 0 \\
$1991-1995$ & 131 & 0 \\
$1996-2000$ & 353 & 2 \\
$2001-2005$ & 726 & 11 \\
$2006-2010$ & 2153 & 132 \\
$2011-2015$ & 3119 & 201 \\
$2016-2018$ & 2.178 & 215 \\
\hline TOTAL & $\mathbf{8 . 7 3 0}$ & $\mathbf{5 6 3}$ \\
\hline
\end{tabular}

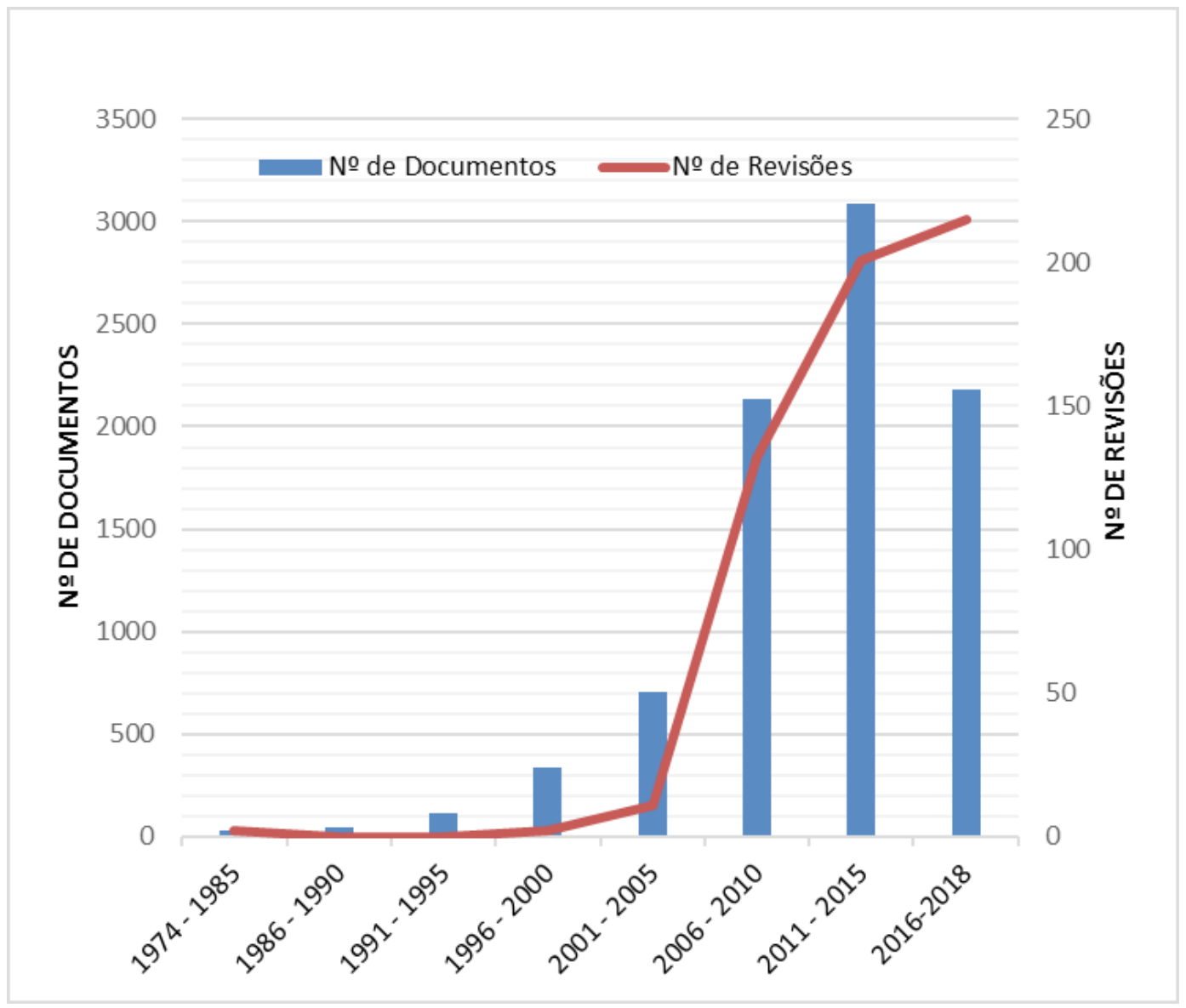

Figura 1: HCPA - crescimento da produção científica no período 1974-2018.

As publicações do HCPA cobrem praticamente todas as áreas da medicina e das ciências biomédicas. A Tabela 3 lista as principais áreas, ou seja, aquelas em que foi publicado um mínimo de 50 artigos. Noutras 104 áreas foram publicados 908 artigos, perfazendo o total de 9.612 publicações no período 1974-2018, aí incluída a dupla-contagem de artigos classificados em mais de uma área. No conjunto, as 46 áreas da Tabela 3 ( $18 \%$ do total de 252 áreas científicas da Web of Science) somam 8.704 publicações, ou seja, $90,5 \%$ do total de documentos, com grande destaque para as áreas de psiquiatria, neurociências, neurologia clínica e comportamento, que juntas respondem por 1.905 artigos $(19,8 \%)$ do total de publicações. 
Guimarães et al.

Tabela 3: Produção científica do Hospital de Clínicas de Porto Alegre - Áreas do conhecimento: 1974-2018.

\begin{tabular}{|c|c|c|}
\hline N. & Área do conhecimento & Artigos \\
\hline 1 & Psychiatry & 760 \\
\hline 2 & Neurosciences & 666 \\
\hline 3 & Endocrinology Metabolism & 526 \\
\hline 4 & Genetics Heredity & 459 \\
\hline 5 & Clinical Neurology & 411 \\
\hline 6 & Medicine Research Experimental & 393 \\
\hline 7 & Pharmacology Pharmacy & 331 \\
\hline 8 & Surgery & 308 \\
\hline 9 & Pediatrics & 302 \\
\hline 10 & Cardiac Cardiovascular Systems & 265 \\
\hline 11 & Oncology & 259 \\
\hline 12 & Obstetrics Gynecology & 239 \\
\hline 13 & Biochemistry Molecular Biology & 236 \\
\hline 14 & Public Environmental Occupational Health & 219 \\
\hline 15 & Medicine General Internal & 214 \\
\hline 16 & Infectious Diseases & 203 \\
\hline 17 & Nutrition Dietetics & 188 \\
\hline 18 & Immunology & 181 \\
\hline 19 & Respiratory System & 168 \\
\hline 20 & Biology & 153 \\
\hline 21 & Multidisciplinary Sciences & 146 \\
\hline 22 & Gastroenterology Hepatology & 140 \\
\hline 23 & Cell Biology & 133 \\
\hline 24 & Peripheral Vascular Disease & 112 \\
\hline 25 & Microbiology & 108 \\
\hline 26 & Pathology & 102 \\
\hline 27 & Medical Laboratory Technology & 99 \\
\hline 28 & Critical Care Medicine & 94 \\
\hline 29 & Transplantation & 93 \\
\hline 30 & Nursing & 92 \\
\hline 31 & Dermatology & 88 \\
\hline 32 & Rheumatology & 88 \\
\hline 33 & Urology Nephrology & 80 \\
\hline 34 & Toxicology & 79 \\
\hline 35 & Sport Sciences & 76 \\
\hline 36 & Otorhinolaryngology & 74 \\
\hline 37 & Reproductive Biology & 73 \\
\hline 38 & Biotechnology Applied Microbiology & 70 \\
\hline 39 & Behavioral Sciences & 68 \\
\hline 40 & Hematology & 68 \\
\hline 41 & Physiology & 67 \\
\hline 42 & Ophthalmology & 65 \\
\hline 43 & Dentistry Oral Surgery Medicine & 52 \\
\hline 44 & Psychology & 52 \\
\hline 45 & Psychology Developmental & 52 \\
\hline \multirow[t]{4}{*}{46} & Radiology Nuclear Medicine Medical Imaging & 52 \\
\hline & Total da Tabela & 8.704 \\
\hline & 104 Outras Áreas & 908 \\
\hline & Total geral* & $9.612^{*}$ \\
\hline
\end{tabular}

* Inclui dupla contagem de artigos. 
A distribuição das palavras-chave e os destaques dos temas de pesquisa do HCPA podem ser visualizados na Figuras 2 e 3, que apresentam, respectivamente, o mapa das palavras mais frequentes nos títulos dos artigos publicados e a lista dos temas descritores desses artigos. Verifica-se nas Figuras a predominância nos títulos dos artigos de palavras-chave (em inglês) como Patients, Disease, Gene, Disorder, Syndrome, Bipolar, Oxidative Stress, Inflammation, Diabetes, Depression, Cancer, Analysis, Schizophrenia, Obesity, Heart Pailure, Epidemiology, Treatment, Clinical, Therapy, e muitas outras que dão uma clara ideia da ampla gama de áreas de pesquisa que caracteriza a atuação dos pesquisadores do HCPA.

A produção científica do HCPA está distribuída em um grande número de periódicos. A Tabela 4 mostra essa dispersão localizada em 1.636 revistas, com 6.630 artigos. Ressalte-se que estes são os números reais de artigos do HCPA no período 19742018, ou seja, sem a dupla-contagem presente nas Tabela 1 e Gráfico 1, onde tal situação se verifica.

Um total de 139 revistas publicou pelo menos 10 artigos do HCPA no período 1974-2018, totalizando 3.093 publicações, ou seja, $46,7 \%$ do total de 6.630 artigos do período. A Tabela 5 apresenta o número de publicações em 24 revistas brasileiras com 10 ou mais artigos do HCPA no período. Do total de 964 artigos nas revistas brasileiras, destacam-se as publicações no Brazilian Journal of Medical and Biological Research, com 124 artigos, ou cerca de $13 \%$ do total nessas revistas.

Assim, do total de publicações das 139 revistas que divulgaram pelo menos 10 artigos cada, 115 são periódicos internacionais. A Tabela 6 apresenta os dados para essas principais revistas que juntas publicaram um total de 2.129 desses artigos. Aqui o destaque é a Plos One, com o maior número (112) artigos.

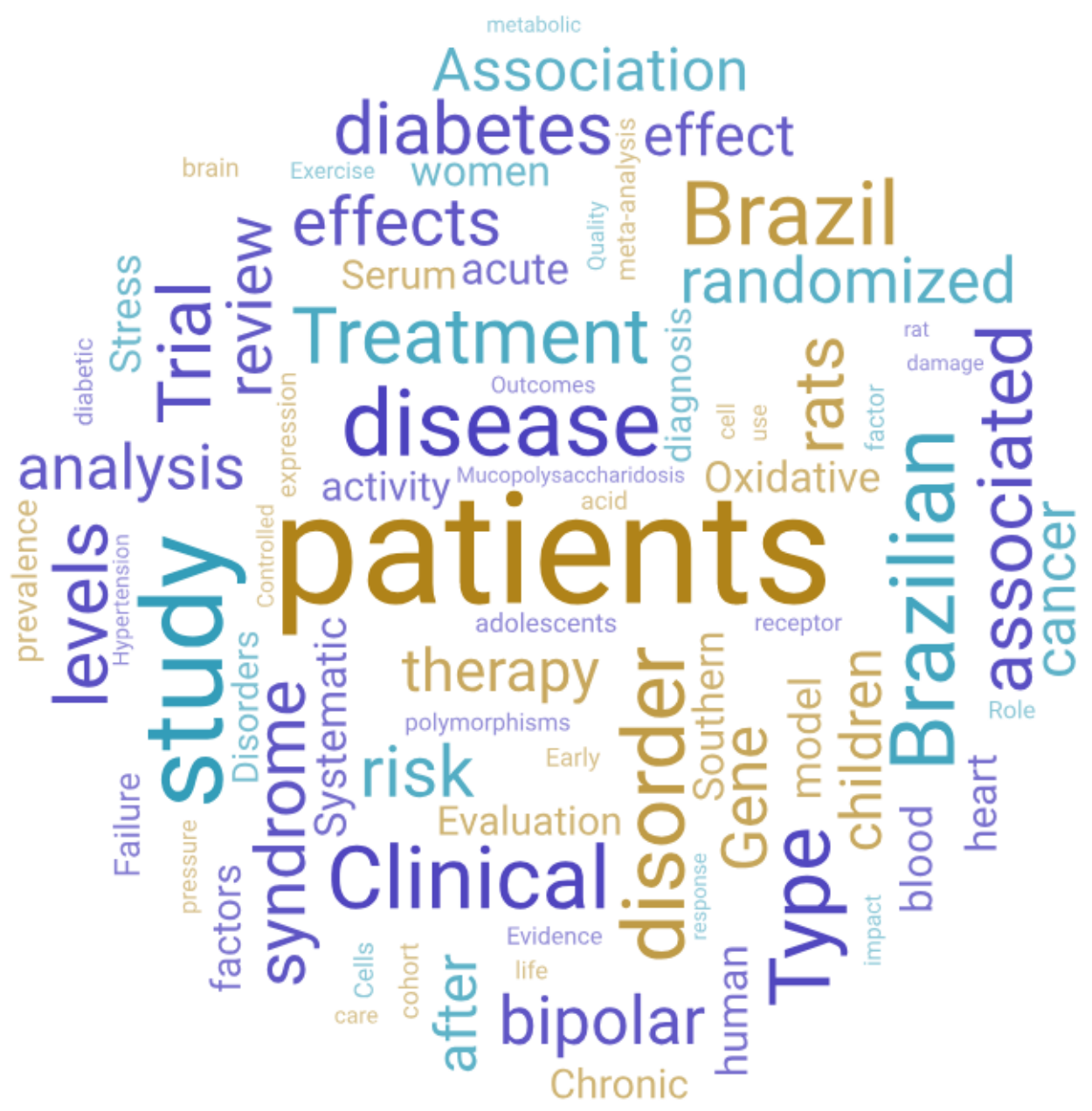

Figura 2: Mapa das palavras-chave dos títulos dos artigos do HCPA indexados na Web of Science: $1974-2018$. 


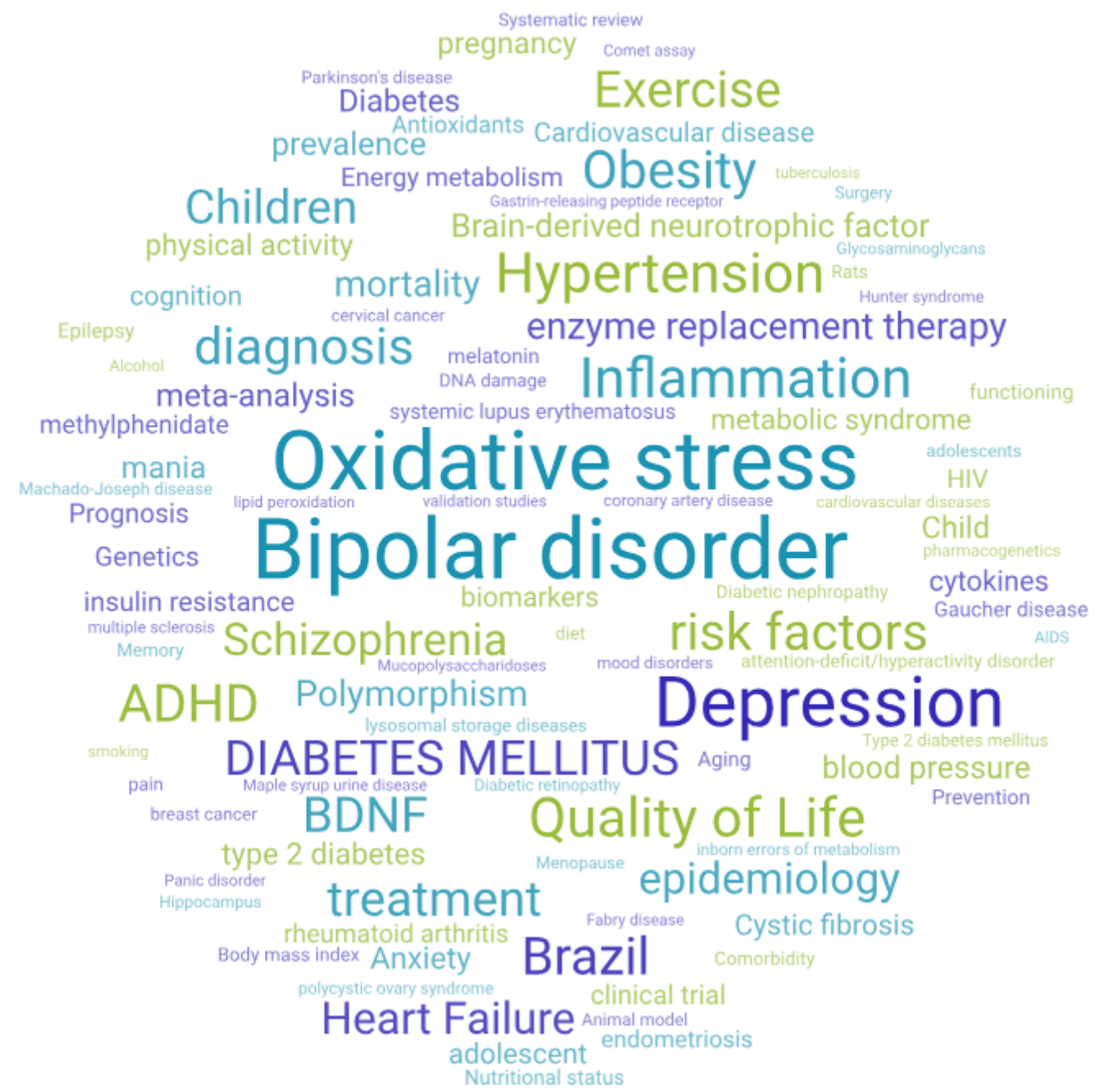

Figura 3: Mapa das palavras-chave (descritores) dos artigos do HCPA indexados na Web of Science: $1974-2018$.

Tabela 4: HCPA: Dispersão das Publicações - 1974 a 2018.

\begin{tabular}{ccc}
\hline Artigos/Revista & N. de revistas & Total de artigos \\
\hline 1 & 735 & 735 \\
2 & 291 & 582 \\
3 & 169 & 507 \\
4 & 98 & 392 \\
5 & 64 & 320 \\
6 & 60 & 360 \\
7 & 20 & 140 \\
8 & 39 & 312 \\
9 & 21 & 189 \\
\hline 10 & 139 & 3.093 \\
\hline TOTAL & 1.636 & 6.630 \\
\hline
\end{tabular}


Tabela 5: HCPA: artigos em revistas brasileiras, 1974-2018.

\begin{tabular}{|c|c|c|}
\hline N. & Revistas brasileiras & Artigos \\
\hline 1 & Brazilian Journal of Medical and Biological Research & 124 \\
\hline 2 & Revista Brasileira de Psiquiatria & 101 \\
\hline 3 & Arquivos Brasileiros de Cardiologia & 77 \\
\hline 4 & Arquivos de Neuro Psiquiatria & 76 \\
\hline 5 & Genetics and Molecular Biology & 71 \\
\hline 6 & Arquivos Brasileiros de Endocrinologia e Metabologia & 67 \\
\hline 7 & Jornal Brasileiro de Pneumologia & 62 \\
\hline 8 & Jornal de Pediatria & 57 \\
\hline 9 & Brazilian Journal of Infectious Diseases & 37 \\
\hline 10 & Clinics & 34 \\
\hline 11 & Revista da Associação Medica Brasileira & 32 \\
\hline 12 & Anais Brasileiros de Dermatologia & 29 \\
\hline 13 & Revista de Saúde Publica & 29 \\
\hline 14 & Acta Cirúrgica Brasileira & 24 \\
\hline 15 & Cadernos de Saúde Publica & 22 \\
\hline 16 & Revista da Escola de Enfermagem da USP & 20 \\
\hline 17 & Arquivos Brasileiros de Oftalmologia & 18 \\
\hline 18 & Abcd - Arquivos Brasileiros de Cirurgia Digestiva & 14 \\
\hline 19 & Brazilian Journal of Otorhinolaryngology & 14 \\
\hline 20 & Revista Brasileira de Reumatologia & 14 \\
\hline 21 & Ciência Saúde Coletiva & 11 \\
\hline 22 & Revista Brasileira de Genética & 11 \\
\hline 23 & Anais da Academia Brasileira de Ciências & 10 \\
\hline \multirow[t]{2}{*}{24} & São Paulo Medical Journal & 10 \\
\hline & TOTAL DA TABELA & 964 \\
\hline
\end{tabular}

Tabela 6: HCPA: artigos em revistas internacionais: 1974-2018.

\begin{tabular}{clc}
\hline N. & \multicolumn{1}{c}{ Revistas internacionais } & Artigos \\
\hline 1 & Plos One & 112 \\
2 & Journal of Affective Disorders & 62 \\
3 & Molecular Genetics and Metabolism & 58 \\
4 & Journal of Psychiatric Research & 53 \\
5 & Journal of Inherited Metabolic Disease & 45 \\
6 & Neuroscience Letters & 44 \\
7 & Diabetology Metabolic Syndrome & 36 \\
8 & Diabetes Care & 35 \\
9 & Transplantation Proceedings & 35 \\
10 & Clinical Biochemistry & 34 \\
11 & Nutricion Hospitalaria & 34 \\
12 & International Journal of Developmental Neuroscience & 33 \\
13 & Metabolic Brain Disease & 33 \\
15 & Clinica Chimica Acta & 32 \\
16 & Diabetes Research and Clinical Practice & 31 \\
17 & Brain Research & 30 \\
18 & Molecular Neurobiology & 28 \\
20 & Neurochemical Research & 27 \\
& Progress in Neuro Psychopharmacology Biological Psychiatry & 27 \\
\end{tabular}


Tabela 6: Continuação

\begin{tabular}{|c|c|c|}
\hline $\mathbf{N}$. & Revistas internacionais & Artigos \\
\hline 21 & Revista Latino Americana de Enfermagem & 27 \\
\hline 22 & European Child Adolescent Psychiatry & 26 \\
\hline 23 & Trials & 25 \\
\hline 24 & Gene & 23 \\
\hline 25 & Lancet & 23 \\
\hline 26 & Scientific Reports & 22 \\
\hline 27 & American Journal of Medical Genetics Part a & 21 \\
\hline 28 & Clinical Genetics & 21 \\
\hline 29 & Acta Psychiatrica Scandinavica & 20 \\
\hline 30 & Cellular and Molecular Neurobiology & 20 \\
\hline 31 & Biomed Research International & 19 \\
\hline 32 & Life Sciences & 19 \\
\hline 33 & Acta Scientiae Veterinariae & 18 \\
\hline 34 & Archives of Endocrinology Metabolism & 18 \\
\hline 35 & Behavioural Brain Research & 18 \\
\hline 36 & Orphanet Journal of Rare Diseases & 18 \\
\hline 37 & Journal of Neural Transmission & 17 \\
\hline 38 & Cerebellum & 16 \\
\hline 39 & Clinical Rheumatology & 16 \\
\hline 40 & International Journal of Cardiology & 16 \\
\hline 41 & Journal of Critical Care & 16 \\
\hline 42 & Mutation Research Genetic Toxicology and Environmental Mutagenesis & 16 \\
\hline 43 & Respiratory Care & 16 \\
\hline 44 & Gynecologic Oncology & 15 \\
\hline 45 & Journal of Clinical Endocrinology Metabolism & 15 \\
\hline 46 & Journal of Endocrinological Investigation & 15 \\
\hline 47 & Journal of Human Hypertension & 15 \\
\hline 48 & Lupus & 15 \\
\hline 49 & Neurochemistry International & 15 \\
\hline 50 & Neuroscience & 15 \\
\hline 51 & Thyroid & 15 \\
\hline 52 & American Journal of Medical Genetics Part b: Neuropsychiatric Genetics & 14 \\
\hline 53 & Biochimica et Biophysica Acta Molecular Basis of Disease & 14 \\
\hline 54 & Bipolar Disorders & 14 \\
\hline 55 & Chronobiology International & 14 \\
\hline 56 & Fertility and Sterility & 14 \\
\hline 57 & Hormone and Metabolic Research & 14 \\
\hline 58 & International Journal of Nursing Knowledge & 14 \\
\hline 59 & Journal of Pediatric Surgery & 14 \\
\hline 60 & Molecular Genetics and Metabolism Reports & 14 \\
\hline 61 & Obesity Surgery & 14 \\
\hline 62 & American Journal of Hypertension & 13 \\
\hline 63 & Comprehensive Psychiatry & 13 \\
\hline 64 & Critical Care & 13 \\
\hline 65 & Digestive Diseases and Sciences & 13 \\
\hline 66 & European Archives of Psychiatry and Clinical Neuroscience & 13 \\
\hline 67 & European Journal of Obstetrics Gynecology and Reproductive Biology & 13 \\
\hline 68 & Journal of Clinical Psychiatry & 13 \\
\hline 69 & Mycopathologia & 13 \\
\hline 70 & Bmc Infectious Diseases & 12 \\
\hline 71 & Clinical Endocrinology & 12 \\
\hline
\end{tabular}


Tabela 6: Continuação

\begin{tabular}{|c|c|c|}
\hline $\mathbf{N}$. & Revistas internacionais & Artigos \\
\hline 72 & Gynecologic and Obstetric Investigation & 12 \\
\hline 73 & Intensive Care Medicine & 12 \\
\hline 74 & International Journal of Gynecological Cancer & 12 \\
\hline 75 & Journal of Hypertension & 12 \\
\hline 76 & Journal of Pediatrics & 12 \\
\hline 77 & Journal of the Neurological Sciences & 12 \\
\hline 78 & New England Journal of Medicine & 12 \\
\hline 79 & Schizophrenia Research & 12 \\
\hline 80 & American Heart Journal & 11 \\
\hline 81 & Annals of Hepatology & 11 \\
\hline 82 & Antimicrobial Agents and Chemotherapy & 11 \\
\hline 83 & Australian and New Zealand Journal of Psychiatry & 11 \\
\hline 84 & Ciencia Saude Coletiva & 11 \\
\hline 85 & Clinical Infectious Diseases & 11 \\
\hline 86 & Clinical Neurophysiology & 11 \\
\hline 87 & Human Reproduction & 11 \\
\hline 88 & Journal of Antimicrobial Chemotherapy & 11 \\
\hline 89 & Journal of Child and Adolescent Psychopharmacology & 11 \\
\hline 90 & Journal of Pediatric Gastroenterology and Nutrition & 11 \\
\hline 91 & Laryngoscope & 11 \\
\hline 92 & Molecular Biology Reports & 11 \\
\hline 93 & Neurotoxicity Research & 11 \\
\hline 94 & Pharmacogenomics & 11 \\
\hline 95 & Value in Health & 11 \\
\hline 96 & Acta Neuropsychiatrica & 10 \\
\hline 97 & American Journal of Infection Control & 10 \\
\hline 98 & Clinical Chemistry and Laboratory Medicine & 10 \\
\hline 99 & Critical Care Medicine & 10 \\
\hline 100 & Endocrine & 10 \\
\hline 101 & Epilepsy Behavior & 10 \\
\hline 102 & European Journal of Endocrinology & 10 \\
\hline 103 & Genetics in Medicine & 10 \\
\hline 104 & Gynecological Endocrinology & 10 \\
\hline 105 & Human Immunology & 10 \\
\hline 106 & International Journal of Neuropsychopharmacology & 10 \\
\hline 107 & International Journal of Pediatric Otorhinolaryngology & 10 \\
\hline 108 & Journal of Cardiac Failure & 10 \\
\hline 109 & Journal of Nervous and Mental Disease & 10 \\
\hline 110 & Latin American Journal of Pharmacy & 10 \\
\hline 111 & Medicine & 10 \\
\hline 112 & Parkinsonism Related Disorders & 10 \\
\hline 113 & Pediatrics & 10 \\
\hline 114 & Renal Failure & 10 \\
\hline 115 & World Journal of Gastroenterology & 10 \\
\hline & \multicolumn{2}{|l|}{ Total da Tabela } \\
\hline
\end{tabular}

A Tabela 7 apresenta uma comparação dos dois grupos de revistas. Os 964 artigos nas revistas brasileiras e os 2.129 nas estrangeiras correspondem, respectivamente, a $31,2 \%$ e $68,8 \%$, do total de 3.093 artigos publicados pelos 139 periódicos científicos. Ou seja, $46,7 \%$ das publicações do HCPA desde 1974 foram publicados nessas revistas, que representam apenas $8,5 \%$ de todas as 1.636 revistas onde os pesquisadores do HCPA publicaram seus artigos ao longo desses 48 anos. Vale destacar na Tabela a alta percentagem de revistas e artigos na área de neurociências: 36 periódicos, sendo 34 internacionais $(30 \%)$ e 873 artigos $(41 \%)$ dos dados dessas revistas. 
Tabela 7: Revistas com 10 ou mais artigos do HCPA: 1974-2018

\begin{tabular}{cccc}
\hline Revista & $\mathbf{N}^{\mathbf{0}}$ & Total de artigos \% Artigos \\
\hline BRASILEIRAS & 24 & 964 & 31,2 \\
Neurociências & 2 & 177 & 19,5 \\
ESTRANGEIRAS & 115 & 2.129 & 68,8 \\
Neurociências & 34 & 873 & 41,0 \\
TOTAIS TABELA & 139 & 3.093 & 100 \\
TOTAL HCPA & 1.636 & 6.630 & ---- \\
\%TABELA/HCPA & 8,5 & 46,7 & ---- \\
\hline
\end{tabular}

As publicações científicas dos pesquisadores do HCPA têm forte presença da colaboração internacional. Os dados da Web of Science indicam um índice de cooperação internacional do HCPA de $44,7 \%$, o qual é bem mais elevado do que a média brasileira, que é da ordem de $29 \%{ }^{5}$ e mesmo da média brasileira para as áreas médicas de $35,5 \%{ }^{3}$. A Tabela 8 lista o número de publicações produzidas em colaboração com 64 principais países, num total de 5.953 artigos, aí incluído um alto índice de dupla-contagem. Outros 678 trabalhos foram ainda produzidos com colaboradores de outros países, perfazendo um total de 6.631 artigos que, somados aos 4.796 originados somente no Brasil, atingem os 11.427 artigos da Tabela 8. Aqui se verifica largo índice de duplacontagem $(72 \%)$ devido à possibilidade de que um mesmo artigo possa incluir, além do autor do HCPA pelo Brasil, endereços de autores de dois ou mais outros países. Tabela 8: HCPA: produção científica em colaboração internacional 1974-2018.

Tabela 8: HCPA: produção científica em colaboração internacional 1974-2018.

\begin{tabular}{|c|c|c|}
\hline N. & País de colaboração & Artigos \\
\hline 1 & USA & 970 \\
\hline 2 & England & 355 \\
\hline 3 & Canada & 323 \\
\hline 4 & Spain & 291 \\
\hline 5 & Australia & 256 \\
\hline 6 & Germany & 242 \\
\hline 7 & Italy & 240 \\
\hline 8 & France & 239 \\
\hline 9 & Argentina & 186 \\
\hline 10 & Portugal & 148 \\
\hline 11 & Belgium & 135 \\
\hline 12 & Netherlands & 133 \\
\hline 13 & Switzerland & 120 \\
\hline 14 & Mexico & 100 \\
\hline 15 & Chile & 86 \\
\hline 16 & Sweden & 86 \\
\hline 17 & Japan & 83 \\
\hline 18 & Colombia & 82 \\
\hline 19 & Poland & 78 \\
\hline 20 & Finland & 77 \\
\hline 21 & India & 72 \\
\hline 22 & Peoples Rep China & 72 \\
\hline 23 & Russia & 71 \\
\hline 24 & Austria & 68 \\
\hline 25 & South Africa & 66 \\
\hline 26 & Taiwan & 65 \\
\hline 27 & Norway & 61 \\
\hline 28 & Denmark & 60 \\
\hline
\end{tabular}


Tabela 8: Continuação

\begin{tabular}{|c|c|c|}
\hline $\mathrm{N}$. & País de colaboração & Artigos \\
\hline 29 & Israel & 56 \\
\hline 30 & Peru & 51 \\
\hline 31 & Turkey & 48 \\
\hline 32 & Greece & 47 \\
\hline 33 & Cuba & 44 \\
\hline 34 & Scotland & 43 \\
\hline 35 & Ireland & 42 \\
\hline 36 & Slovakia & 42 \\
\hline 37 & Uruguay & 42 \\
\hline 38 & Romania & 40 \\
\hline 39 & South Korea & 40 \\
\hline 40 & Hungary & 37 \\
\hline 41 & Philippines & 35 \\
\hline 42 & Saudi Arabia & 35 \\
\hline 43 & Czech Republic & 34 \\
\hline 44 & Malaysia & 33 \\
\hline 45 & Costa Rica & 32 \\
\hline 46 & Singapore & 32 \\
\hline 47 & Thailand & 32 \\
\hline 48 & New Zealand & 31 \\
\hline 49 & Serbia & 31 \\
\hline 50 & Slovenia & 29 \\
\hline 51 & Uganda & 29 \\
\hline 52 & Venezuela & 28 \\
\hline 53 & Nigeria & 27 \\
\hline 54 & Vietnam & 27 \\
\hline 55 & Egypt & 25 \\
\hline 56 & Iran & 25 \\
\hline 57 & Kenya & 24 \\
\hline 58 & Pakistan & 23 \\
\hline 59 & Qatar & 23 \\
\hline 60 & Croatia & 21 \\
\hline 61 & Bangladesh & 20 \\
\hline 62 & Ecuador & 20 \\
\hline 63 & Ghana & 20 \\
\hline \multirow[t]{5}{*}{64} & Jordan & 20 \\
\hline & Outros países & 678 \\
\hline & Total em colaboração internacional & 6.631 \\
\hline & Brasil sem colaboração internacional & 4.796 \\
\hline & Total geral* & 11.427 \\
\hline
\end{tabular}

*Inclui dupla contagem de artigos. 
Destaca-se na Tabela a presença majoritária da colaboração com autores dos Estados Unidos da América, com 970 artigos, seguido de Inglaterra (355), Canadá (323), Espanha (291), Austrália (256), Alemanha (242), Itália (240) e França (239). Juntos esses oito países correspondem a $26 \%$ dos artigos em colaboração internacional do HCPA. Não é, pois, sem razão que vários dos artigos mais citados provêm dessas colaborações.

Neste sentido, uma análise mais detalhada na base Web of Science dos dados da produção científica do HCPA permite identificar os artigos com maior número de citações. Foram identificados 50 desses artigos com destaque nesse indicador, em sua maioria (48) publicados entre 2001 e 2016. Confirmando a observação anterior sobre a presença de forte índice de colaboração internacional nas publicações dos pesquisadores do hospital (Tabela 6), verifica-se aqui que uma larga proporção (39/50) desses artigos de elevado número de citações resulta de artigos de colaboração científica internacional. Apesar de serem relativamente recentes, estes artigos apresentam elevados índices de citações, variando de 127 a 2.295, este último de um artigo mais antigo (1995). Tal situação não é incomum, pois se sabe que a cooperação internacional, especialmente com autores dos países mais desenvolvidos, implica em maior número de citações e, por consequência, na elevação dos indicadores qualitativos como o índice de impacto dos artigos 5 .

Destaque-se, como situação ímpar, que um artigo de 2016, sobre Zika Virus e microcefalia, recebeu, até 2018 , um total de 212 citações, uma situação bastante incomum para um artigo tão recente.

A produção científica do HCPA tem a participação de um grande número de pesquisadores, com vínculo com o Hospital, com a Faculdade de Medicina e outras unidades da UFRGS. Vale alertar aqui que vários autores, porque têm uma vínculo empregatício com a Faculdade de Medicina ou outra unidade da UFRGS, com frequência não indicam nas publicações sua filiação ao HCPA, ainda que o trabalho tenha sido feito em seus laboratórios. Tais artigos foram identificados como tendo endereço HCPA pela presença entre os autores, de colaboradores vinculados diretamente ao hospital.

\section{CONSIDERAÇÕES FINAIS}

Em conclusão, os dados aqui apresentados relativos à produção científica do HCPA mostram uma trajetória positiva dessa instituição ao longo de seus mais de 48 anos, que, ao lado de sua reconhecida competência como hospital modelo, tem destacada atuação na produção de novos conhecimentos científicos por meio da pesquisa básica e clínica - o que, de resto, alicerça sua qualificação médicohospitalar. Para efeito conclusivo desse exercício cientométrico, restaria a recomendação ao GPPG de que os pesquisadores que atuam na pesquisa no Hospital fizessem a indicação do HCPA nos seus artigos, mesmo quando vinculados a Faculdade de Medicina ou outras unidades da UFRGS.

\section{Referências}

1. Hospital de Clínicas de Porto Alegre. Hospital de Clínicas de Porto Alegre, uma referência em saúde: relatório anual 2009. Porto Alegre: HCPA; 2009.

2. Bones E. Hospital de Clínicas de Porto Alegre - Parte 1: na região dos sonhos. Porto Alegre: Backstage; 2013.
3. Clarivates Analytics [Internet]. 2019 [citado 2019 Ago 7]. Disponível em: https://clarivate.com/

4. Almeida ECE, Guimarães JA. Brazil's growing production of scientific articles - how are we doing with review articles and other qualitative indicators? Scientometrics. 2013;97(2):287-315.
5. Zanotto SR, Haeffner C, Guimarães JA. Unbalanced international collaboration affects adversely the usefulness of countries' scientific output as well as their technological and social impact. Scientometrics. 2016;109(3):1789-1814. 